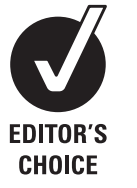

- Additional material is published online only. To view this file please visit the journa online (http://jmg.bmj.com).

${ }^{1}$ Department of Medical Genetics, Montreal Children's Hospital, McGill University,

Montreal, Quebec, Canada ${ }^{2}$ Department of Human Genetics, McGill University, Montreal, Quebec, Canada ${ }^{3}$ Department of Paediatrics, McGill University, Montreal, Quebec, Canada

${ }^{4}$ McGill University-Montreal Children's Hospital Research Institute, Montreal, Quebec, Canada

\section{Correspondence to}

Dr Nancy Braverman, McGill University-Montreal Children's Hospital Research Institute, 4060 Ste-Catherine West, PT-406.2, Montreal, OC H3Z 2Z3, Canada

nancy.braverman@mcgill.ca

Received 5 June 2011 Revised 11 July 2011 Accepted 12 July 2011 Published Online First 23 July 2011

\title{
Combined malonic and methylmalonic aciduria: exome sequencing reveals mutations in the ACSF3 gene in patients with a non-classic phenotype
}

\author{
Ahmed Alfares, ${ }^{1,2}$ Laura Dempsey Nunez, ${ }^{2}$ Khalid Al-Thihlii, ${ }^{1,2}$ John Mitchell, ${ }^{3}$ \\ Serge Melançon, ${ }^{1,2}$ Natascia Anastasio, ${ }^{2}$ Kevin C H Ha, ${ }^{2}$ Jacek Majewski, ${ }^{1,2}$ \\ David S Rosenblatt, ${ }^{1,2,3}$ Nancy Braverman 2,3,4
}

\begin{abstract}
Background Combined Malonic and Methylmalonic Aciduria (CMAMMA) is a rare recessive inborn error of metabolism characterised by elevations of urine malonic acid (MA) and methylmalonic acid (MMA). Nearly all reported cases are caused by malonyl-CoA decarboxylase (MCD) deficiency. Most patients have metabolic acidosis, developmental delay, seizures and cardiomyopathy. CMAMMA was also described in symptomatic patients with normal MCD activity, suggesting heterogeneity in this disorder.
\end{abstract}

Methods and results We identified two probands with a non-classical CMAMMA variant through the Quebec newborn urine screening program. While they share the biochemical phenotype of elevated MA and MMA, the MMA excretion was higher than MA, the clinical courses were benign, MYLCD gene sequencing was normal and MCD activity, measured in one proband, was normal. Using exome sequencing in the single consanguineous proband, we identified a homozygous missense allele in the ACSF3 gene, encoding an Acyl-CoA Synthetase (ACS) with unknown substrate and function. The second proband was homozygous for a different ACSF3 missense allele. Both substitutions were in conserved residues and were identified in less than $0.5 \%$ of their respective ethnic control populations.

Conclusion These results suggest that ACSF3 is a candidate gene for non-classical CMAMMA observed in our patients and document the value of exome sequencing of a limited number of patients for the identification of novel disease genes.

Several types of patients with elevated malonic acid (MA) have been described in the literature. The most well characterised is that related to malonylcoenzyme A (CoA) decarboxylase (MCD) enzyme deficiency (OMIM \#248360) in which the biochemical abnormalities include elevated $\mathrm{MA}$ alone, or combined elevations of MA and methylmalonic acid (CMAMMA) with MA mainly being higher than methylmalonic acid (MMA). ${ }^{1-11}$ It is speculated that MCD deficiency causes an excess of intramitochondrial malonyl-CoA, leading to the inhibition of methylmalonyl-CoA mutase and, subsequently, an increase in MMA. Two patients have been described with similar combined elevations of MA greater than MMA, but with normal MCD enzyme activity in fibroblast cultures ${ }^{12} 13$ suggesting that this disorder is heterogeneous. Al of these patients have shown a variety of clinical features that include metabolic acidosis, developmental delay, seizures, cardiomyopathy, gastrointestinal distress and dysmorphic features, although normal development was also reported. ${ }^{11}$ Through the Quebec newborn urine screening programme ${ }^{14}$ we identified asymptomatic individuals with CMAMMA but with MMA higher than MA, and sought to define the underlying molecular cause. We refer to these patients as having non-classic CMAMMA.

\section{METHODS}

\section{Metabolite detection}

The Quebec newborn urine screening programme, including MMA since 1975, evaluates urine from 4-6-week-old infants by thin layer chromatography. Subsequent urine organic acid analysis by gas chromatography mass spectroscopy confirms CMAMMA. MMA is considered elevated when there is more than $200 \mu \mathrm{mol} / \mathrm{mmol}$ creatinine in the newborn period. ${ }^{15}$ Patients were followed in our medical genetics clinic and informed consent for research studies was obtained according to institutional guidelines.

\section{Molecular analysis}

Genomic DNA was isolated from patient fibroblast cell lines. All exons and intronic flanking sequences of MLYCD were amplified and sequenced by Sanger methods; primers and PCR conditions were modified from Wightman et al. ${ }^{11}$ ACSF3 exons 5 and 8 were amplified from gDNA in 315 controls using primers and conditions reported here (see supplementary material, available online only). GenBank ref seq nos: MLYCD, NM_012213; ACSF3, NM_174917; TAS2R43, NM_176884.

\section{Exome sequencing}

A total of $3 \mu \mathrm{g}$ of DNA was subjected to the SureSelect Human All Exon v2 Kit from Agilent Technologies (Santa Clara, CA, USA), according to the manufacturer's protocols. This kit targets approximately $44 \mathrm{Mb}$ of sequence including the human exome defined by the NCBI consensus coding sequence database (version 20090902) and additional ref seq sequences. The captured DNA was sequenced by Illumina GAIIx (San Diego, CA, USA) on one lane, generating approximately 32 million 
76 bp quality-controlled reads. Reads were aligned to the human reference genome (University of California, Santa Cruz (UCSC) hg19 assembly) using Burrows-Wheeler Aligner's SmithWaterman Alignment (BWA). ${ }^{16}$ Reads that mapped nonuniquely or to unfinished chromosomes were discarded from further analysis.

Approximately 28.9 million reads were uniquely mapped to the reference genome, providing an average NCBI consensus coding sequence coverage of $25 \mathrm{X}$. We imposed similar variant identification filtering criteria as described. ${ }^{17}$ Variants were identified using SAMtools. ${ }^{16}$ In addition, we compared our variants against a pool of 34 exomes previously sequenced by us, of which three were reported ${ }^{17} 18$ while the remainder are unpublished. Variants seen in two or more individuals were discarded. Functional annotation of the predicted variants was carried out using ANNOVAR, ${ }^{19}$ which cross-references variants against public databases, including dbSNP131 and the 1000 Genomes project. Novel variants were defined as those absent from dbSNP and had a 1000 Genomes allele frequency of less than 0.005 . This allowed us to consider variants observed in 1000 Genomes at very rare frequencies. Potentially damaging variants included non-synonymous substitutions caused by missense and nonsense single-nucleotide polymorphisms (SNP), splice-site SNP, and frameshift changes due to indels.

\section{RESULTS}

The clinical and biochemical features of the two non-classic CMAMMA probands in this report are described below.

Patient 1, a 14-year-old boy, was born to non-consanguineous Ashkenazi Jewish parents (figure 1A, II:3) and was briefly reported previously. ${ }^{15}$ His initial urine MMA level was $891 \mu \mathrm{mol} / \mathrm{mmol}$ creatinine. Confirmatory testing by gas chromatography mass spectroscopy showed a urine MMA of $745 \mu \mathrm{mol} / \mathrm{mmol}$ creatinine (normal $<25$ ) and MA of $60 \mu \mathrm{mol} /$ mmol creatinine (normal $<6$ ). His plasma MMA level was $18 \mu \mathrm{mol} / \mathrm{l}$ (normal <0.5). On follow-up the ranges of urine MA and MMA were $20-68$ and $214-470 \mu \mathrm{mol} / \mathrm{mmol}$ creatinine, respectively; the plasma MMA range was $20-32 \mu \mathrm{M}$.

Patient 2, a 4-year-old girl, was born to first cousin French Canadian parents (figure 1B, II:1). Her initial MMA level was $800 \mu \mathrm{mol} / \mathrm{mmol}$ creatinine, and confirmatory testing showed urine MMA and MA levels of 737 and $67 \mu \mathrm{mol} / \mathrm{mmol}$ creatinine, respectively; the plasma MMA level was $39.2 \mu \mathrm{mol} / \mathrm{l}$. On followup the ranges of urine MA and MMA were 43-260 and 690-1830 $\mu \mathrm{mol} / \mathrm{mmol}$ creatinine, respectively. The range of plasma MMA was $28-40 \mu \mathrm{M}$. She has a 2-year-old similarly affected sibling (figure 1B, II:2) with urine MMA 843 and MA $94 \mu \mathrm{mol} / \mathrm{mmol}$ creatinine.

Both patients were born at term, were clinically asymptomatic, had normal cardiac examinations and age-appropriate development. No other organic acid abnormality was detected. Carnitine levels, acylcarnitine profiles, plasma amino acids, ammonia, blood gas values, total homocysteine, folate and vitamin $B_{12}$ levels were all within reference ranges, and the mothers were not vitamin $B_{12}$ deficient. MMA levels were not responsive to protein restriction or vitamin $B_{12}$ supplementation and therefore these treatments were not instituted. In cultured fibroblasts, the incorporation of $\left[{ }^{14} \mathrm{C}\right]$-propionate, $\left[{ }^{14} \mathrm{C}\right]$-methyltetrahydrofolate and $\left[{ }^{57} \mathrm{C}\right]$-cyanocobalamin, and the synthesis of both adenosylcobalamin and methylcobalamin were within the reference range. These results excluded a defect in intracellular vitamin $\mathrm{B}_{12}$ metabolism and methylmalonyl CoA deficiency. MCD activity in fibroblasts was measured in patient 1 and the results were within the reference range.

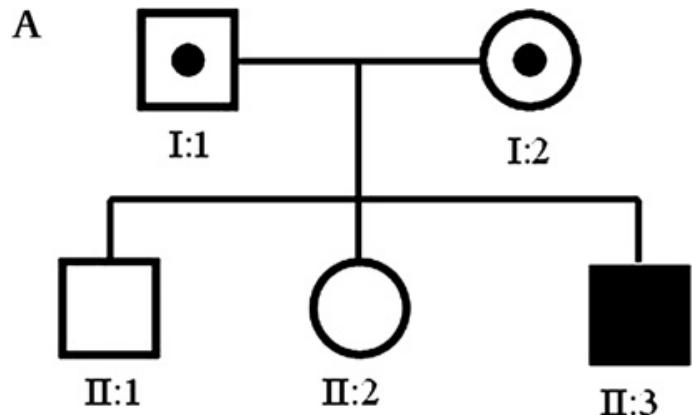

\section{Molecular analysis}

No mutations in MYLCD were found in either patient. Exome sequencing of patient 2, followed by the selection of novel non-synonymous variants (see Methods) that were either homozygous or potentially compound heterozygous (two mutations in the same gene) resulted in the identification of only two potential candidate genes: TAS2R43 (taste receptor, type 2, member 43) and ACSF3, a novel member of the family of acyl-CoA synthetases (ACS). TAS2R 43 encodes a seven transmembrane G-protein-coupled receptor located on the surface of taste receptor cells and mediates cell responses to bitter taste. ${ }^{20}$ A homozygous missense substitution in a residue conserved among mammals, c.97A $\rightarrow \mathrm{G}$ (p.Ile33Val), was identified and predicted to be located in a potential cytoplasmic domain. We did not detect any region of homozygosity around this variant. However, based on potential biological significance, ACSF3 was selected as the more likely candidate. Patient 2 was homozygous for c.1075G $\rightarrow$ A in exon 5, predicted to substitute a glutamate to a lysine residue, or p.Glu359Lys. The glutamate residue is highly conserved through evolution among all ACSF3 homologues, including zebrafish, neurospora and rice, and this substitution was predicted to be damaging based on PolyPhen-2 functional analysis. ${ }^{21}$ This allele was annotated in 1000 Genomes with a rare allele frequency of 0.001 . There were no heterozygous SNP in the vicinity of this mutation, suggesting a region of homozygosity spanning approximately $5.4 \mathrm{MB}$ at the tail of chromosome 16q. This mutation was also identified in homozygosity in the affected brother. In order to determine whether ACSF3 play a role in the patients' phenotype, ACSF3 was sequenced in patient 1 , and a homozygous mutation, c.1411C $\rightarrow \mathrm{T}$ (p.Arg471Trp), was identified in exon 8. The arginine residue is again highly conserved in evolution and the substitution was predicted to be damaging. ${ }^{21}$ The Sanger 
sequence traces for both mutations and parental genotypes are shown in figure 2.

To determine the frequency of the missense alleles in an ethnically matched population, we sequenced ACSF3 exon 5 in 139 French Canadian controls and exon 8 in 176 Ashkenazi Jewish controls. The c.1075G $\rightarrow$ A (exon 5) mutation was found in a heterozygous state in one French Canadian control individual, predicting a minimum incidence of this phenotype to be one in 77284 in this population. The c.1411C $\rightarrow \mathrm{T}$ (exon 8) mutation was found in a heterozygous state in a single Ashkenazi Jewish control individual, predicting a minimum incidence of this phenotype to be one in 123094 .

\section{DISCUSSION}

CMAMMA is a rare recessive phenotype characterised by elevated MA and MMA in body fluids. The non-classic patients described here differ from classic patients by having a benign clinical course. When the urine MA levels are compared they overlap; the ranges of MA are approximately 15-260 in nonclassic and $19-600 \mu \mathrm{mol} / \mathrm{mmol}$ creatinine in classic patients. However, the MMA levels in non-classic patients range from approximately 400 to $1000 \mu \mathrm{mol} / \mathrm{mmol}$ creatinine, which is higher than the classic range of $5-300 \mu \mathrm{mol} / \mathrm{mmol}$. The detection of these patients may be unique to the Quebec newborn urine screening programme. Most newborn screening programmes analyse dried blood spots using tandem mass spectrometry in the first week of life. Infants with classic CMAMMA are suspected by elevations of C3-DC (malonyl) acylcarnitine. It is not known whether non-classic CMAMMA can be detected by this method, considering that the targeted C3-DC, C3 and C4-DC plasma acylcarnitine species were within reference ranges in our patients.

The number of newborns screened per year in Quebec is approximately 77000 , and ethnicity is not determined (Dr Christiane Auray-Blais, Faculty of Medicine and Health Sciences, Université de Sherbrooke, personal communication). Therefore, approximately 2695000 infants were screened from 1975 to 2010 and three patients with non-classic CMAMMA were detected. This detection rate of one per 898333 is lower than that predicted by the heterozygote frequencies. These figures indicate that not all newborns with this biochemical phenotype are detected by the Quebec urine screening programme and further support the clinical evidence that this may be a benign phenotype.

ACSF3 contains nine coding exons and is located on chromosome 16q24.3. There are two transcript variants that differ by the presence of an alternate exon in the $5^{\prime}$ untranslated region. EST profiles show transcipt expression in numerous tissues and organs. ACSF 3 encodes a 576 amino acid protein containing a predicted $\mathrm{N}$-terminal mitochondrial targeting signal. It is a member of the family of ACS, which catalyse the activation of fatty acids by forming a thioester with CoA and a fatty acid, to form acyl-CoA. Recently, 26 distinct ACS were identified in the human genome based on the presence of five conserved amino acid motifs. ${ }^{22}$ ACSF 3 could not be assigned to any of the established subfamilies (ACSS, ACSM, ACSL, ACSVL, or ACSBG and probably represented a novel ACS family member. Watkins et al ${ }^{22}$ demonstrated that ACSF3 did indeed have ACS activity in a heterologous expression system. p.Arg471 located in ACS motif II and mutated in patient 1, represents an ATP binding site by homology and is nearly invariant in all ACS families. Substitution of the equivalent residue in Escherichia coli fatty ACS to an alanine (fadD, p.Arg453Ala) resulted in the complete loss of enzymatic activity. ${ }^{23}$

We suggest that defects in ACSF3 are good candidates for the biochemical phenotype in these patients. Future studies will need to be performed to determine whether ACSF3 deficiency is causative of the non-classic CMAMMA described here. Given a putative role as a mitochondrial ACS enzyme, we speculate that ACSF3 might encode a malonyl or methylmalony-CoA synthetase. Previous studies have suggested the possibility of a methylmalony-CoA synthetase providing a 'free shunt' for MMA in the mitochondria. ${ }^{24}$ For malonylCoA synthetase, it is interesting to note that the ACSF3 protein has $35 \%$ identity (amino acids $48-573$ ) with bacterial malonyl-CoA synthetases using position-specific iterated BLASTp algorithms. ${ }^{25}$

Exome sequencing is a method to identify novel disease genes using one or a few affected individuals. ${ }^{1726} 27$ In this case, the

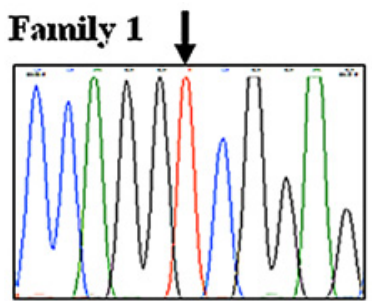

Patient 1, II:3 c. $1411 \mathrm{C} \rightarrow \mathrm{T}$

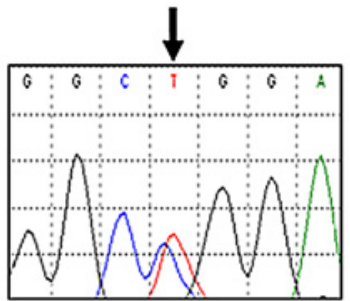

Father, heterozygous at c.1411

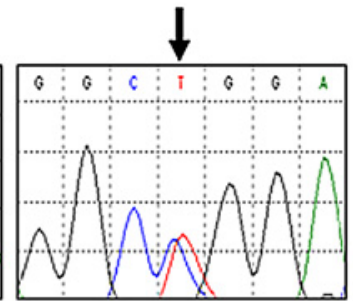

Mother, heterozygous at c.1411

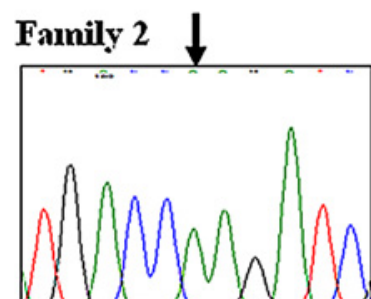

Patient 2, II:1 c. $1075 \mathrm{G} \rightarrow \mathrm{A}$

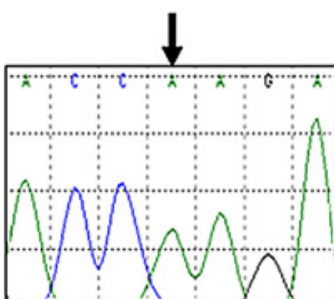

Brother II:2 c. $1075 \mathrm{G} \rightarrow \mathrm{A}$

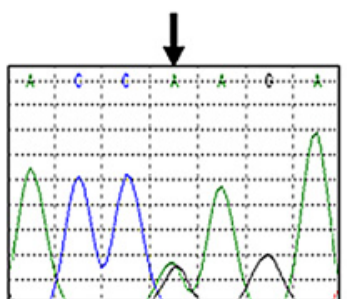

Father, heterozygous at c. 1075

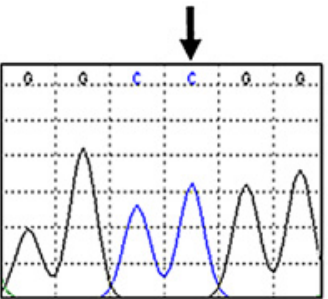

Control

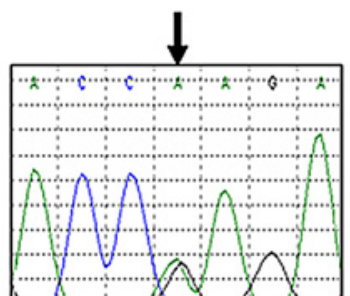

Mother, heterozygous at c. 1075

Figure 2 Sanger sequence traces in the mutation region for patients 1, 2, parents and control. 
finding of a homozygous missense allele in ACSF3, facilitated by consanguinity, was corroborated by finding a different homozygous missense allele in the same gene in a second proband. This highlights the growing power of this technique to find novel candidate disease genes with limited numbers of patients and families.

Acknowledgements The authors acknowledge the contributions of the families in these research endeavours.

Funding Funding was received from the Montreal Children's Hospital Research Institute (NB), from the Canadian Institutes for Health Research (DSR and JM) and a Canada Research Chair (JM). DSR and NB are members of the Research Institute of the McGill University Health Centre, which is supported in part by the Fonds de la Recherche en Santé (Quebec)

\section{Competing interests None.}

Ethics approval Ethics approval was obtained from Montreal Children's Hospital and MUHC.

Contributors $\mathrm{AA}, \mathrm{NB}, \mathrm{KAT}, \mathrm{JM}$ and SM identified and characterised the patients: $\mathrm{KHH}$ and JM performed the exome sequencing and analysis; LDN, NA and DSR performed the biochemical and molecular studies; AA and NB wrote the paper; LDN, $\mathrm{DSR}, \mathrm{KHH}$ and JM contributed to the final manuscript; NB is the guarantor.

Provenance and peer review Not commissioned; externally peer reviewed.

\section{REFERENCES}

1. Brown GK, Scholem RD, Bankier A, Danks DM. Malonyl coenzyme A decarboxylase deficiency. J Inherit Metab Dis 1984;7:21-6.

2. Matalon R, Michaels K, Kaul R, Whitman V, Rodriguez-Novo J, Goodman S, Thorburn D. Malonic aciduria and cardiomyopathy. J Inherit Metab Dis 1993:16:571-3

3. MacPhee GB, Logan RW, Mitchell JS, Howells DW, Tsotsis E, Thorburn DR. Malonyl coenzyme A decarboxylase deficiency. Arch Dis Child 1993;69:433-6.

4. Krawinkel MB, Oldigs HD, Santer R, Lehnert W, Wendel U, Schaub J. Association of malonyl-CoA decarboxylase deficiency and heterozygote state for haemoglobin $\mathrm{C}$ disease. J Inherit Metab Dis 1994;17:636-7.

5. Haan EA, Scholem RD, Croll HB, Brown GK. Malonyl coenzyme A decarboxylase deficiency. Clinical and biochemical findings in a second child with a more severe enzyme defect. Eur J Pediatr 1986;144:567-70.

6. Gao J, Waber L, Bennett MJ, Gibson KM, Cohen JC. Cloning and mutational analysis of human malonyl-coenzyme A decarboxylase. J Lipid Res 1999;40:178-82.

7. Buyukgebiz B, Jakobs C, Scholte HR, Huijmans JG, Kleijer WJ. Fatal neonatal malonic aciduria. J Inherit Metab Dis 1998;21:76-7.

8. Yano S, Sweetman L, Thorburn DR, Mofidi S, Williams JC. A new case of malonyl coenzyme A decarboxylase deficiency presenting with cardiomyopathy. Eur J Pediatr 1997;156:382-3.

9. Salomons GS, Jakobs C, Pope LL, Errami A, Potter M, Nowaczyk M, Olpin S, Manning N, Raiman JAJ, Slade T, Champion MP, Peck D, Gavrilov D, Hillman R, Hoganson GE, Donaldson K, Shield JPH, Ketteridge D, Wasserstein M, Gibson KM. Clinical, enzymatic and molecular characterization of nine new patients with malonylcoenzyme A decarboxylase deficiency. J Inherit Metab Dis 2007;30:23-8.
10. Malvagia S, Papi L, Morrone A, Donati MA, Ciani F, Pasquini E, la Marca G, Scholte HR, Genuardi M, Zammarchi E. Fatal malonyl CoA decarboxylase deficiency due to maternal uniparental isodisomy of the telomeric end of chromosome 16. Ann Hum Genet 2007:71:705-12.

11. Wightman PJ, Santer R, Ribes A, Dougherty F, McGill N, Thorburn DR, FitzPatrick DR. MLYCD mutation analysis: evidence for protein mistargeting as a cause of MLYCD deficiency. Hum Mutat 2003;22:288-300.

12. Gregg AR, Warman AW, Thorburn DR, O'Brien WE. Combined malonic and methylmalonic aciduria with normal malonyl-coenzyme A decarboxylase activity: a case supporting multiple aetiologies. J Inherit Metab Dis 1998;21:382-90.

13. Ozand PT, Nyhan WL, al Aqeel A, Christodoulou J. Malonic aciduria. Brain Dev 1994;16(Suppl):7-11.

14. Auray-Blais C, Giguère R, Lemieux B. Newborn urine screening programme in the province of Quebec: an update of 30 years' experience. J Inherit Metab Dis 2003:26:393-402.

15. Sniderman LC, Lambert M, Giguère R, Auray-Blais C, Lemieux B, Laframboise R, Rosenblatt DS, Treacy EP. Outcome of individuals with low-moderate methylmalonic aciduria detected through a neonatal screening program. J Pediatr 1999; 134:675-80.

16. Li H, Durbin R. Fast and accurate short read alignment with Burrows-Wheeler transform. Bioinformatics 2009;25:1754-60.

17. Lalonde E, Albrecht S, Ha KCH, Jacob K, Bolduc N, Polychronakos C, Dechelotte P, Majewski J, Jabado N. Unexpected allelic heterogeneity and spectrum of mutations in Fowler syndrome revealed by next-generation exome sequencing. Hum Mutat 2010;31:918-23

18. Anastasio N, Ben-Omran T, Teebi A, Ha KCH, Lalonde E, Ali R, Almureikhi M, Der Kaloustian VM, Liu J, Rosenblatt DS, Majewski J, Jerome-Majewska LA. Mutations in SCARF2 are responsible for Van Den Ende-Gupta syndrome. Am J Hum Genet 2010;87:553-9.

19. Wang K, Li M, Hakonarson H. ANNOVAR: functional annotation of genetic variants from high-throughput sequencing data. Nucleic Acids Res 2010;38:e164.

20. Kinnamon SC. Taste receptor signalling-from tongues to lungs. Acta Physiol (Oxf) Published Online First: 11 April 2011. doi:10.1111/j.1748-1716.2011.02308.x

21. Adzhubei IA, Schmidt S, Peshkin L, Ramensky VE, Gerasimova A, Bork P, Kondrashov AS, Sunyaev SR. A method and server for predicting damaging missense mutations. Nat Methods 2010;7:248-9.

22. Watkins PA, Maiguel D, Jia Z, Pevsner J. Evidence for 26 distinct acylcoenzyme A synthetase genes in the human genome. J Lipid Res 2007;48 2736-50.

23. Black PN, Zhang 0, Weimar JD, DiRusso CC. Mutational analysis of a fatty acylcoenzyme A synthetase signature motif identifies seven amino acid residues that modulate fatty acid substrate specificity. J Biol Chem 1997;272:4896-903.

24. Montgomery JA, Mamer OA, Scriver CR. Metabolism of methylmalonic acid in rats Is methylmalonyl-coenzyme a racemase deficiency symptomatic in man? J Clin Invest 1983; 72:1937-47.

25. Altschul SF, Madden TL, Schäffer AA, Zhang J, Zhang Z, Miller W, Lipman DJ. Gapped BLAST and PSI-BLAST: a new generation of protein database search programs. Nucleic Acids Res 1997;25:3389-402.

26. Ng SB, Turner EH, Robertson PD, Flygare SD, Bigham AW, Lee C, Shaffer T, Wong M Bhattacharjee A, Eichler EE, Bamshad M, Nickerson DA, Shendure J. Targeted capture and massively parallel sequencing of 12 human exomes. Nature 2009;461:272-6

27. Choi M, Scholl UI, Ji W, Liu T, Tikhonova IR, Zumbo P, Nayir A, Bakkaloğlu A, Ozen S, Sanjad S, Nelson-Williams C, Farhi A, Mane S, Lifton RP. Genetic diagnosis by whole exome capture and massively parallel DNA sequencing. Proc Natl Acad Sci U S A 2009:106:19096-101. 